\title{
Immunohistochemical detection of FSH receptors in pituitary adenomas and adrenal tumors
}

\author{
Marek Pawlikowski ${ }^{1}$, Hanna Pisarek ${ }^{2}$, Robert Kubiak ${ }^{3}$, \\ Maria Jaranowska ${ }^{2}$, Henryk Stępień ${ }^{1}$ \\ ${ }^{1}$ Department of Immunoendocrinology, $1^{\text {st }}$ Chair of Endocrinology, Medical University of Lodz, Poland \\ ${ }^{2}$ Department of Neuroendocrinology, Chair of Laboratory Medicine, \\ Medical University of Lodz, Poland \\ ${ }^{3}$ Department of Pathology of Tumors, Chair of Oncology, Medical University of Lodz, Poland
}

\begin{abstract}
Follicle stimulating hormone (FSH) receptors (FSHR) are physiologically expressed in the ovary and testis. It is well known that FSHR are also expressed in gonadal cancers, but data regarding their incidence in extra-gonadal tumors is scarce. Recently, the expression of FSHR in the vascular endothelium within different human cancers was found, but nothing is yet known about FSHR appearance in non-gonadal endocrine tumors. The present paper reports on the immunohistochemical detection of FSHR in human pituitary adenomas and adrenal tumors. The study included samples of 28 pituitary adenomas and 37 adrenal tumors. Moreover, two samples of non-tumoral adrenal glands were also studied. FSH receptor immunostaining was performed on paraffin sections using the rabbit anti-human FSH-R polyclonal antibody raised against 1-190 amino acid sequence from the human FSH-R (sc-13935). The pituitary adenomas were immunostained to reveal the pituitary hormones and the proliferation marker Ki-67. In the pituitary adenomas, positive immunostaining with anti-FSHR antibody occurred in the adenoma cells cytoplasm and endothelia of the intra- and peritumoral blood vessels. Cytoplasmic immunostaining was found in the majority of investigated tumors, but the intensity of staining was weak to moderate. There was some tendency towards a higher cytoplasmic FSHR score in tumors with higher Ki-67 index (atypical adenomas). In contrast to the cytoplasm, the FSHR immunostaining in blood vessels was strong and concerned all the investigated samples. Strong FSHR immunostaining was present in the endothelium of intra- and/or peritumoral blood vessels in the majority of pheochromocytomas, approximately one half of the adrenocortical adenomas, and all cases of adrenal cancers. The immunostaining was detectable also in the tumoral cell cytoplasm in all but one examined pheochromocytomas. All the investigated adrenocortical adenomas presented strong immunostaining of cell membranes. No immunostained cell membranes were found in adrenal cancers. The positive immunostaining was found in glandular cells, but not in blood vessels of non-tumoral adrenal cortex and medulla. Immunostaining of FSHR often occurs in the endothelium of intra- and/or peritumoral blood vessels of pituitary adenomas and benign and malignant adrenal tumors. The immunostaining may be also present in neoplastic cells. A role of FSHR expression in these tumors, such as stimulation of angiogenesis or of cell proliferation, needs to be clarified in further studies. (Folia Histochemica et Cytobiologica 2012, Vol. 50, No. 3, 325-330)
\end{abstract}

Key words: FSH receptors, pituitary adenoma, adrenocortical adenoma, adrenal cancer, pheochromocytoma, vascular endothelium

Correspondence address: M. Pawlikowski, Department of Immunoendocrinology, $1^{\text {st }}$ Chair of Endocrinology,

Medical University of Lodz,

Dr Sterling Str. 3, 91-425 Lodz, Poland;

tel./fax: + 48426365427 ;

e-mail: marek.pawlikowski@umed.lodz.pl

\section{Introduction}

FSH receptors (FSHR) are membrane receptors transducing the biological signal of the follicle stimulating hormone (follitropin, FSH) on its target cells. FSHR are expressed in normal conditions in female 
and male gonads; in the granulosa cells of the ovary and in Sertoli cells of the testis. They are known also to be expressed in gonadal tumors, both in males and females [for review see: 1]. Less is known about FSHR expression in extra-gonadal tumors. Several tumors have been shown to express luteinizing hormone/ /chorionic gonadotrophin receptors (LH/CG-R) and the tumorigenic action of $\mathrm{LH}$ and chorionic gonadotropin (CG) has been hypothesized [1].

The expression of FSHR has been investigated by means of polymerase chain reaction (PCR) and immunohistochemistry in the human prostate and prostate cancers. The presence of FSHR in the normal prostate and benign prostate hyperplasia has been found, but the expression was higher in prostate cancer. In prostate cancer cell lines, FSHR were present in an androgen resistant line PC3 but absent in androgen-sensitive LNCaP cells [2]. Recently, Radu et al. [3], using immunohistochemistry and in situ hybridization, have reported the expression of FSHR in intratumoral and peritumoral blood vessels in a large series of human cancers, including prostate, breast, colon, pancreas, urinary bladder, kidney, lung, liver, stomach, testis and ovary. To the best of our knowledge, FSHR expression has not been studied to date in extra-gonadal endocrine tumors. The present paper shows that FSHR are expressed also in pituitary adenomas and adrenal tumors.

\section{Material and methods}

Tumor samples. Twenty eight pituitary adenomas and 37 adrenal tumors, surgically excised, and one post mortem collected hyperplastic adrenal gland, were included in the study.

The pituitary adenomas included five samples from patients with acromegaly, two prolactinomas, 17 clinically nonfunctioning adenomas, and four corticotropinomas manifesting as Cushing's disease. In the group of clinically nonfunctioning pituitary adenomas (CNFPA), seven expressed gonadotrophins, three expressed ACTH without the symptoms of Cushing's disease ('silent' corticotropinomas), two expressed growth hormone without the symptoms of acromegaly ('silent' somatotropinomas), and five were plurihormonal (they expressed GH/PRL/LH in two cases, $\alpha$-subunit/GH/PRL in one case, $\mathrm{ACTH} / \mathrm{LH}$ in one case and GH/ /LH in one case). The adrenal tumors included eight pheochromocytomas, 26 adrenocortical adenomas and three adrenal cancers. Within the adrenocortical adenomas, four were excised in patients suffering from Conn's syndrome and four in patients with Cushing's syndrome; the remaining 16 were nonfunctioning. Moreover, one sample of adrenocortical hyperplasia from a female patient with ACTH-dependent Cushing's syndrome due to the ectopic ACTH secretion by a neuroendocrine thymic tumor and a sample of non-tumoral adrenal cortex adjacent to pheochromocytoma tumor was also examined.

Immunohistochemistry. FSH receptor immunostaining was performed using the rabbit anti-human FSH-R polyclonal antibody sc-13935. This antibody was raised against 1-190 amino acid sequence from the human FSH-R. The antibody was purchased from Santa Cruz Biotechnology Inc. (Santa Cruz, CA, USA) and its specificity had been approved in earlier publications $[4,5]$. The antibody was applied in a working dilution of 1:100. Each pituitary adenoma was previously immunostained using the antibodies against the pituitary hormones and alpha-subunit (alpha-SU). More detailed information on pituitary hormone immunohistochemistry was published in an earlier paper [6]. The hormone immunohistochemistry, taken together with clinical data, served to classify the pituitary adenoma samples. Additionally, 16 pituitary adenomas were also immunostained with anti-Ki-67 (MIB-1) antibody (Dako Cytomation, Glostrup, Denmark). The visualization of primary antibodies was done using either the StreptABComplex/HRP Duet (Dako Cytomation, pituitary hormones and Ki-67) or the Dako REAL EnVision Detection System (FSHR) following the procedures recommended by the manufacturer. The intensity of the immunoreaction for FSHR protein in the tumoral cells cytoplasm was scored semiquantitatively using a descriptive scale as follows: negative staining ( ing ( score $=1$ ), moderate staining (score $=2$ ) and strong staining (score $=3$ ). The statistical comparison of scores was performed by means of Student's $t$ test. For a positive control, a biopsy sample of the human testis was immunostained. For a negative control, the primary antibody was omitted in the staining procedure.

The study was approved by the Local Bioethical Committee, decision number RNN/537/11/KB dated 14 June 2011.

\section{Results}

\section{Pituitary adenomas}

In pituitary adenomas, positive immunostaining with anti-FSHR antibody occurred in adenoma cells cytoplasm and endothelia of the intra- and peritumoral blood vessels. Cytoplasmic immunostaining was found in the majority of investigated tumors $(25 / 28)$ but the intensity of staining was either weak (score $=1$ ) or moderate (score $=2$, Figure 1 ). There was no appreciable difference between primary and recurrent pituitary adenomas (mean scores \pm SEM, $1.1 \pm 0.15$ and $1.25 \pm 0.28$, respectively). However, the higher score of FSHR in tumors with Ki-67 index $>3$ in comparison to those with $\mathrm{Ki}-67<3$ was found (mean scores \pm SEM, $1.2 \pm 0.2$ and $1.8 \pm 0.3$, respectively, $\mathrm{p}<0.05)$. Our material was too scarce to relate the FSHR expression in the tumoral cells cytoplasm with 
the hormonal phenotype of the investigated adenomas. In contrast to the cytoplasm, the FSHR immunostaining in blood vessels is strong and concerned all the investigated samples (Figure 2). However, in $13 / 28$ of examined tumors, the immunostaining of the endothelium was fragmentary, present in some but not all vessels, and/or present only in limited areas of the endothelium.

\section{Pheochromocytomas}

Strong FSHR immunostaining was present in the endothelium of intra- and/or peritumoral blood vessels in 5/8 pheochromocytomas (Figure 3). The immunostaining was detectable also in tumoral cell cytoplasm in all but one examined pheochromocytomas (7/8, Figure 4). The cytoplasmic FSHR immunopositivity was stronger than that observed in pituitary adenomas, but comparable with that observed in the sample of non-tumoral adrenal medulla and in three fragments of the adrenal medulla adjacent to the adrenocortical tumors.

\section{Adrenocortical adenomas}

FSHR immunostaining was present in the endothelium of intra- and/or peritumoral blood vessels in 12/22 examined tumors (Figure 5). However, the immunopositivity of the endothelium was mostly fragmentary, present in some but not all vessels, and/or present only in limited areas of the endothelium. There was no difference between nonfunctioning and functioning adenomas: the incidence of endothelial immunostaining was $7 / 14$ and 5/8, respectively. All the investigated adrenocortical adenomas presented strong immunostaining of cell membranes. Weak diffuse cytoplasmic staining was also present.
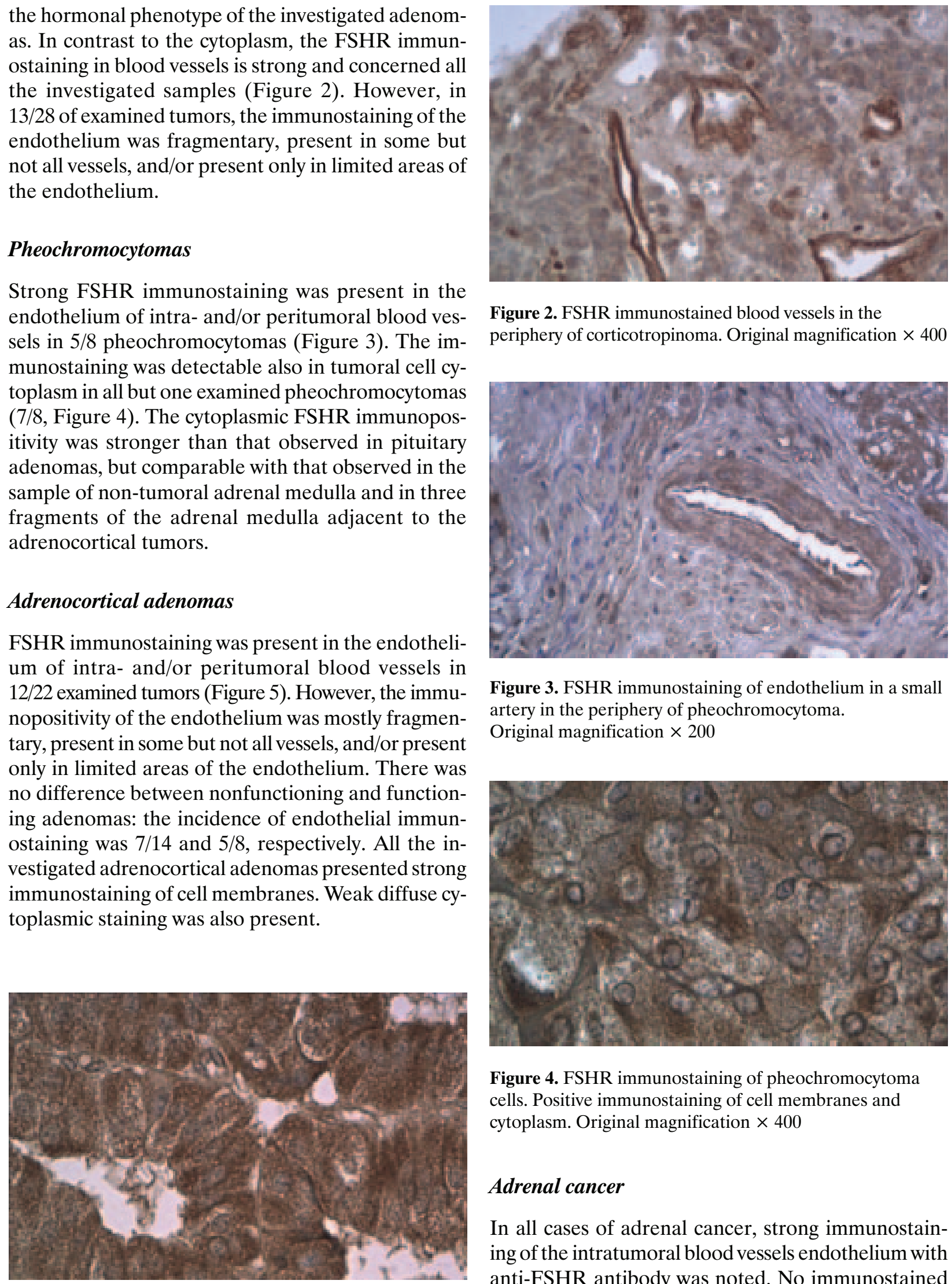

Figure 2. FSHR immunostained blood vessels in the periphery of corticotropinoma. Original magnification $\times 400$

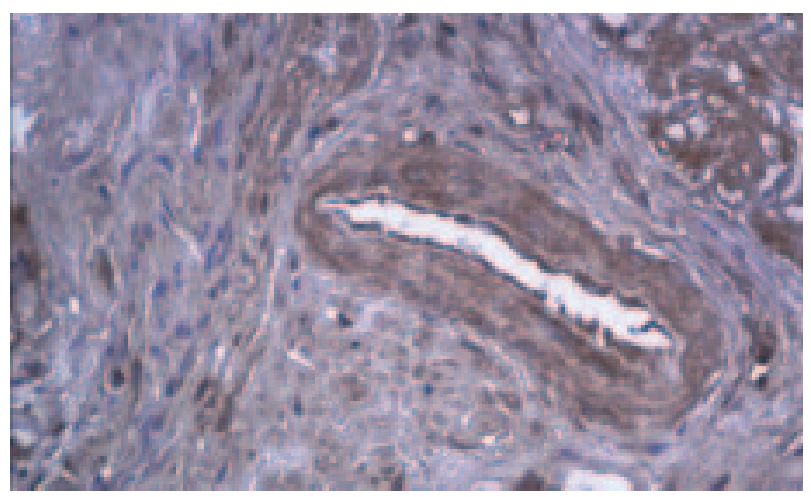

Figure 3. FSHR immunostaining of endothelium in a small artery in the periphery of pheochromocytoma.

Original magnification $\times 200$

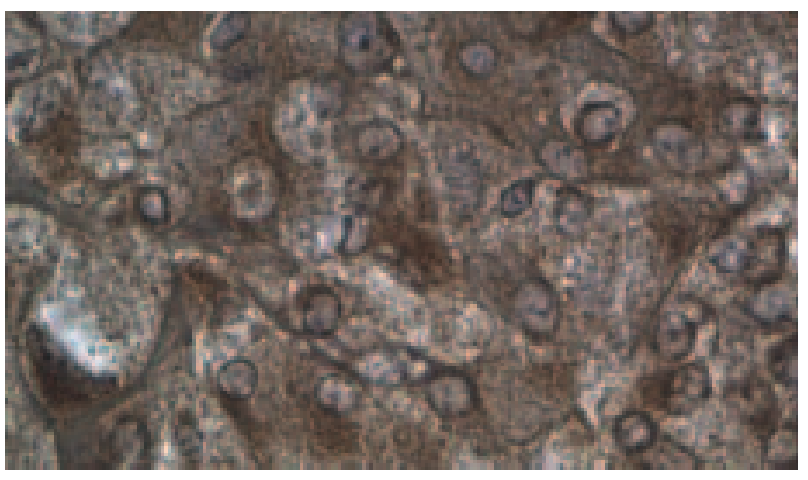

Figure 4. FSHR immunostaining of pheochromocytoma cells. Positive immunostaining of cell membranes and cytoplasm. Original magnification $\times 400$

\section{Adrenal cancer}

In all cases of adrenal cancer, strong immunostaining of the intratumoral blood vessels endothelium with anti-FSHR antibody was noted. No immunostained cell membranes were found. On the other hand, in the cancer cells cytoplasm in two cases the FSHR immunostaining was weak (score $=1$ ) or moderate 


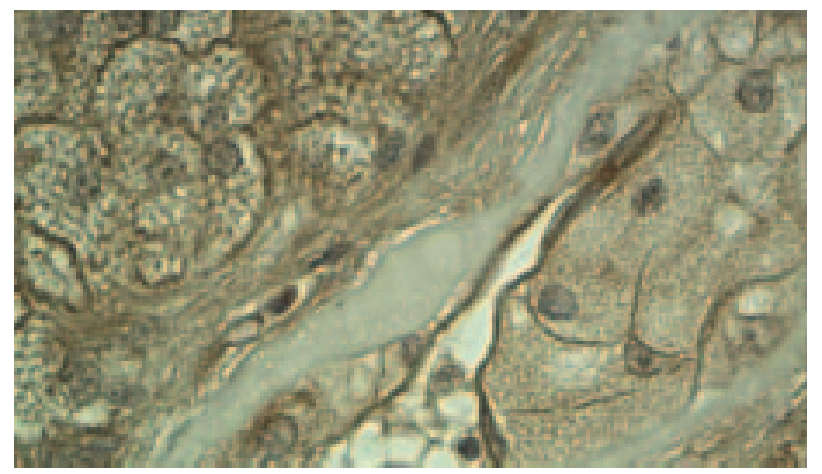

Figure 5. Nonfunctioning adrenocortical adenoma. Strong FSHR immunostaining of adenoma cell membranes and vascular endothelium and weak immunostaining of adenoma cell cytoplasm. Original magnification $\times 400$

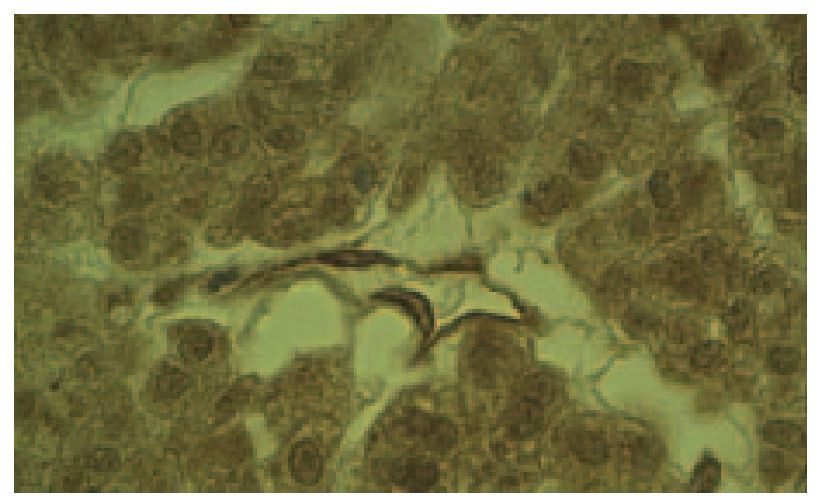

Figure 6. FSHR immunostaining in cytoplasm of tumor cells and vascular wall in the adrenal cancer.

Original magnification $\times 400$

(score $=2$ ), while in the remaining case it was strong (score $=3$, Figure 6 ).

\section{Non-tumoral adrenal cortex and medulla}

In both samples of non-tumoral adrenal cortex (one sample from ectopic Cushing's syndrome and one adjacent to a pheochromocytoma), a strong immunostaining with anti-FSHR antibody localized in adrenocortical cell membranes was found. On the other hand, the cytoplasmic staining was negative or weak ( $($ core $=1)$. In contrast, in the adrenal medulla the immunostaining was only cytoplasmic but strong (score $=3$, Figure 7). The blood vessels were mostly immunonegative. Interestingly, moderate FSHR immunostaining was noticed in the cytoplasm of sympathetic gangliocytes localized in the adrenal gland capsule (Figure 8).

\section{Discussion}

The results presented above show, for the first time, that FSHR expression is detectable by immunohis-

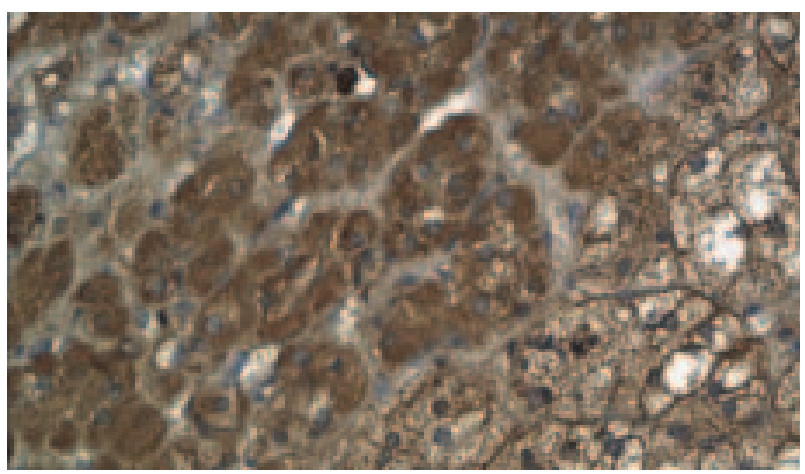

Figure 7. Fragment of non-tumoral adrenal cortex and medulla adjacent to pheochromocytoma tumor. Strong immunostaining of medullar cells (left) and weaker immunostaining of cortical cells (right). Original magnification $\times 200$

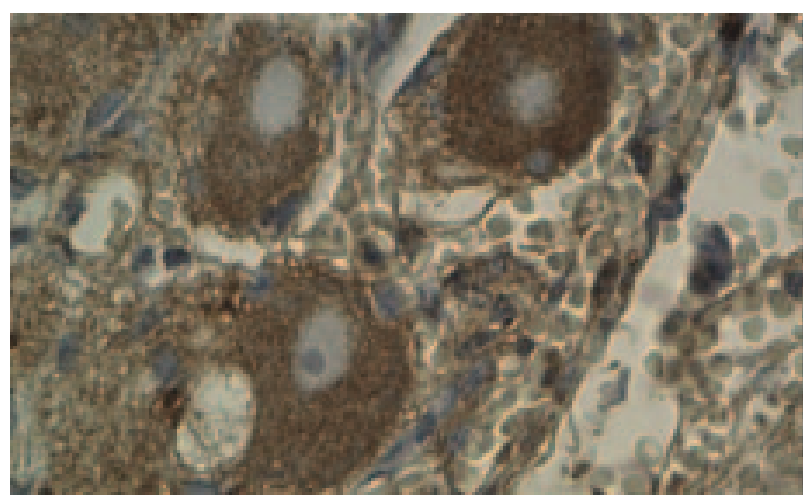

Figure 8. Gangliocytes situated in the adrenocortical capsule. Immunostaining of perikarya can be seen. Original magnification $\times 400$

tochemistry in the intra- and peritumoral blood vessels endothelia in the pituitary adenomas and adrenal tumors. This finding is compatible with the earlier data of Radu et al. [3], who made a similar observation in human cancers of different origins. In contrast to cancers examined by the quoted authors, pituitary adenomas and adrenocortical adenomas are benign tumors. Nevertheless, some pituitary adenomas may exhibit aggressive behavior, consisting in the infiltration of the adjacent structures (invasiveness) and high growth potential. Taken together, our findings show that the expression of FSHR in blood vessels is not correlated with the degree of tumor malignancy. Radu et al. [3] showed that FSHR expression was confined to peri- and intratumoral blood vessels, but was absent in non-tumoral tissue samples, except gonads and placenta. The lack of FSHR immunopositivity within the vessels of non-tumoral adrenal cortex corroborate with the data cited above. On the other hand, FSHR immunostaining in the investigated tumors was not confined to the vascular endothelia, but appeared in the tumoral cells as well. In pituitary 
adenomas, the cytoplasmic FSHR immunostaining seems to be related to the tumor growth potential. Interestingly, the FSHR expression was also found in the non-tumoral adrenal cortex and medulla. The question as to whether the normal adrenal gland is a direct target for gonadotrophins remains unanswered. The direct effects of both FSH and LH were described as long ago as the 1960s [7-9]. The synergic action of FSH and ACTH on adrenal cortex growth was found in hypophysectomized-gonadectomized rats [8]. FSH was also shown to stimulate directly adrenal regeneration in rats, whereas LH inhibited this process [9]. More recently, LH-dependent adrenal tumorigenesis has been demonstrated in transgenic mice expressing a viral oncogene [10]. Moreover, the aberrant expression of $\mathrm{LH} / \mathrm{CG}$ receptors (but not of FSHR) in the adrenal cortex may be responsible for ACTH-independent macronodular adrenal hyperplasia manifesting as Cushing's syndrome [11]. An accidental observation reported in the present paper, that the anti-FSHR antibody reacted with the non-tumoral adrenomedullar cells and peri-adrenal sympathetic gangliocytes, is also interesting because this could be linked with neurovascular disturbances accompanying perimenopausal period in women, characterized by high FSH levels. However, this finding needs further studies to be relevant. We here recall an early paper from our laboratory showing the degenerative changes in sympathetic ganglia after FSH administration in hypohysectomized-gonadectomized rats [12]. If FSHR expressed in endocrine and other tumors are functional, they may exhibit a similar action to that occurring in normal gonads and gonadal tumors. It is known that FSH, acting via its receptors, stimulates the proliferation of ovarian granulosa cells and increases vascular endothelial growth factor (VEGF) secretion within the ovary, leading to enhanced angiogenesis [13]. A similar observation was made with ovarian cancer cells SK-OV-3) [14]. FSH also inhibits ovarian cancer cell apoptosis by up-regulating survivin and down-regulating programmed cell death gene 6 (PDCD6) and death receptor 5 (DR5) [15].

The formation of new blood vessels (angiogenesis) is a process determining the development of solid tumors. VEGF, a specific mitogen of the endothelial cells, belongs to the crucial factors involved in the mechanism of angiogenesis. The inhibition of VEGF expression or inhibition of its receptors has been suggested as a promising way of treating tumors [16]. VEGF levels have been found to be elevated in the blood of patients with pituitary tumors [17-19] and adrenal tumors [20]. In pituitary adenomas, VEGF receptors are expressed not only in endothelial cells of intratumoral blood vessels, but also in tumoral cells [21]. It has been suggested that VEGF in pituitary adenomas acts not only as a stimulator of angiogenesis, but directly promotes the growth of tumoral cells.

\section{Conclusion}

Immunostaining of FSHR was found in the endothelium of intra- and/or peritumoral blood vessels of pituitary adenomas and benign adrenal tumors (adrenocortical adenomas), as well as in potentially malignant (pheochromocytomas) and malignant (cancers) adrenal tumors. The immunostaining may be also present in neoplastic cells. The role of FSHR expression in these tumors, such as stimulation of angiogenesis or growth of tumor cells, needs further studies to be clarified.

\section{Acknowledgement}

This work was supported by European Co-operation in the Field of Scientific and Technical Research COST ACTION grant No CM0602, and Ministry of Science and Higher Education of Poland grant No 505-04-001.

\section{References}

1. Huhtaniemi I. Are gonadotrophins tumorigenic - a critical review of clinical and experimental data. Mol. Cell Endocrinol. 2010;329:56-61.

2. Mariani S, Salvatori L, Basciani S et al. Expression and cellular localization of follicle stimulating hormone receptor in normal human prostate, benign prostatic hyperplasia and prostate cancer. J Urol. 2006;175:2072-2077.

3. Radu A, Pichon C, Camparo P et al. Expression of follicle stimulating hormone receptor in tumor blood vessels. New England J Med. 2010;363:1621-1630.

4. Chuang CK, Lee KH, Fan CT et al. FSH-sensitive murine Sertoli cell lines immortalized by human telomerase gene hTERT express the androgen receptor in response to TNF-alpha stimulation. Biosc Rep. 2007;27:403-411.

5. Bucay N, Yebra M, Cirulli V et al. A novel approach for the derivative of putative primordial germ cells from human embryonic stem cells Stem Cells. 2009;27:68-77.

6. Pawlikowski M, Gruszka A, Kurnatowska I et al. Proliferating cell nuclear antigen (PCNA) expression in pituitary adenomas: relationship to the endocrine phenotype of adenoma. Folia Histochem Cytobiol. 2006;44:37-41.

7. Mikolajczyk H, Pawlikowski T. Histologic changes in the adrenal cortex of hypophysectomized-gonadectomized rats treated with gonadotrophins and adrenocrticotrophin. Endokrynol Pol. 1965;16:359-369.

8. Mikolajczyk H. Possible synergic influence of ACTH and FSH on the adrenal cortex in hypophysectomized-gonadectomized rats. Nature. 1967;213:806-807.

9. Zieleniewski J. The influence of tropic hormones on the regeneration of suprarenal glans In Sprague-Dawley rats [In polish]. Endokrynol Pol. 1968;19:357-381.

10. Vuoremoja S, Rivero-Muller A, Kiiveri S et al. Adrenocortical tumorigenesis, luteinizing hormone receptor and transcrip- 
tion factors GATA-4 and GATA-6. Mol Cell Endocrinol. 2007;269:38-45.

11. Christopoulos S, Bourdeau I, Lacroix A. Aberrant expression of hormone receptors In adrenal Cushing's syndrome Pituitary. 2004;7:225-235.

12. Pawlikowski M. The effect of gonadal and gonadotropic hormones on the prostatic ganglion and superior cervical ganglion in male white rats Acta Medica Polona. 1962;3:171-183.

13. Kuo S, Ke FC, Chang GD et al. Potential role of follicle stimulating hormone (FSH) and transforming growth factor (TGF1) in the regulation of ovarian angiogenesis.J Cell Physiol. 2011;226:1608-1619.

14. Park YH, Kim SJ, Jeong BH et al. Follicular stimulating hormone enhances Notch1 expression in SK-OV-3 ovarian cancer cells. J Gynecol Oncol. 2010;21:119-124.

15. Huang Y, Jin H, Liou I et al. FSH inhibits ovarian cancer cell apoptosis by up-regulating survivin and down regulating PDCD6 and DR5. Endocr Relat Cancer. 2010;18:13-26.

16. Folkman J. Angiogenesis: an organizing principle of drug discovery? Nature Rev. 2007;6:273-285.
17. Komorowski J, Jankiewicz J, Stepień H. Vascular endothelial growth factor (VEGF), basic fibroblast growth factor (bFGF) and soluble interleukin-2 receptor (sIL-2R) concentrations in peripheral blood as markers of pituitary tumors. Cytobios. 2000;101:151-159.

18. Turner HE, Nagy ZS, Gatter KC et al. Angiogenesis in pituitary adenomas and the normal pituitary gland. J Clin Endocrinol Metab. 2000;85:1159-1162.

19. Gruszka A, Kunert-Radek J, Pawlikowski M, Stepien H. Serum endostatin levels are elevated and correlate with serum vascular endothelial growth factor levels in patients with pituitary adenomas. Pituitary. 2005;8:163-168.

20. Jurczynska J, Stepien T, Lawnicka H et al. Peripheral blood concentrations of vascular endothelial growth factor and its soluble receptors (R1 and R2) in patients with adrenal cortex tumours treated by surgery. Endokrynol Pol - Polish J Endocrinol. 2009;60:9-13.

21. Onofri C, Theodoropoulou M, Losa M et al. Localization of vascular endothelial growth factor (VEGF) receptors in normal and adenomatous pituitaries: detection of a non-endothelial function of VEGF in pituitary tumours. J Endocrinol. 2006;191:249-261.

Submitted: 20 February, 2012 Accepted after reviews: 23 July, 2012 